\title{
Identification of Experimental Unsteady Aerodynamic Impulse Responses
}

\author{
Walter A. Silva*, David J. Piatak ${ }^{\dagger}$, and Robert C. Scott ${ }^{\ddagger}$ \\ NASA Langley Research Center \\ Hampton, Virginia 23681-0001
}

\begin{abstract}
The identification of experimental unsteady aerodynamic impulse responses using the Oscillating Turntable (OTT) at NASA Langley's Transonic Dynamics Tunnel (TDT) is described. Results are presented for two configurations: a Rigid Semispan Model (RSM) and a rectangular wing with a supercritical airfoil section. Both models were used to acquire unsteady pressure data due to pitching oscillations on the OTT. A deconvolution scheme involving a step input in pitch and the resultant step response in pressure, for several pressure transducers, is used to identify the pressure impulse responses. The identified impulse responses are then used to predict the pressure response due to pitching oscillations at several frequencies. Comparisons with the experimental data are presented.
\end{abstract}

\section{Introduction}

$\mathrm{C}$ YOMPLEX flight dynamic and aeroelastic phenomena are best understood by studying the underlying unsteady aerodynamic behavior. To this end, experiments designed to measure the unsteady aerodynamic response of various configurations provide significant and valuable information. ${ }^{1-4}$ Experimental results are compared to various types of numerical analyses (such as CFD) to provide insight into the underlying physics of the problem.

Insight gained from unsteady aerodynamic experiments and analyses can then be used to alter or control some aspect of the underlying physics leading to a modification of the performance of a vehicle. This is the primary goal of the flow control research effort. ${ }^{5-8}$ A thorough understanding of the dominant flow physics can lead to an optimal flow control strategy. Therefore, understanding unsteady aerodynamic behavior is an essential step in the design of flow control concepts as well.

For computational methods, the development of reduced-order models (ROM) has proven beneficial towards understanding dominant flow physics. ${ }^{9,10}$ These methods provide insight regarding the level of nonlinearity within a physical process including nonlinear aeroelastic responses and aeroelastic limit cycle oscillations (LCO). In previous work, aerodynamic im-

\footnotetext{
* Senior Research Scientist, Aeroelasticity Branch, NASA Langley Research Center, Hampton, Virginia

${ }^{\dagger}$ Aerospace Engineer, Aeroelasticity Branch, NASA Langley Research Center,Hampton, Virginia

†Senior Aerospace Engineer, Aeroelasticity Branch, NASA Langley Research Center,Hampton, Virginia

Copyright (C) 2003 by the American Institute of Aeronautics and Astronautics, Inc. No copyright is asserted in the United States under Title 17, U.S. Code. The U.S. Government has a royaltyfree license to exercise all rights under the copyright claimed herein for Governmental Purposes. All other rights are reserved by the copyright owner.
}

pulse responses are numerically identified and used to provide insight into the dominant flow physics. These aerodynamic impulse responses are then used to develop computationally-efficient state-space models of the aeroelastic system.

The measurement of unsteady aerodynamic and aeroelastic data is essential to the understanding of the flow physics and structural dynamics that govern a particular experiment. The analysis and interpretation of this type of data, however, is typically hampered by two complications: 1) a wide range of behavior that is difficult to classify as either linear or nonlinear; and 2) noise in the data. The method presented herein targets these complications directly. Although the results presented are for unsteady aerodynamic pressures, the method can be applied to unsteady aerodynamic and aeroelastic loads as well.

The goals of this paper are: 1) to demonstrate the feasibility of identifying unsteady aerodynamic impulse responses from experimental unsteady aerodynamic measurements; and 2) to investigate the filtering capability of the methodology used to generate the aerodynamic impulse responses. The identification of the unsteady aerodynamic impulse responses will provide a direct method for evaluating the linearity (or nonlinearity) of any response. This evaluation is performed by comparing the predicted response using a linearized impulse response with the measured response. The level of correlation between the two responses (predicted versus measured) can be used to define linear and nonlinear regions of response. In addition, the use of a deconvolution technique will provide valuable filtering of the data above and beyond that of standard filters. That is, deconvolution, by definition, generates an impulse response via the correlation of an input/output pair. Data that is not input/output correlated is automatically filtered from 
the creation of the impulse response. Therefore, any uncorrelated white noise that may be present in the measurements will be filtered out via the deconvolution procedure. Note that this filtering capability will not be based on a given frequency range (low-pass, high-pass, or band-pass) but rather on the correlation of the input/output measurements.

This paper begins with a description of the experimental facilities including the Transonic Dynamics Tunnel (TDT), the Oscillating Turntable (OTT) and the wind-tunnel models used to acquire the data. A description of the methodology is then provided, followed by results and finally, concluding remarks.

\section{Description of Experimental Facilities and Models}

\section{Transonic Dynamics Tunnel (TDT)}

The Langley Transonic Dynamics Tunnel (TDT) is a unique national facility dedicated to identifying, understanding, and solving aeroelastic problems. The TDT is a closed-circuit, continuous-flow, variablepressure, wind tunnel with a 16-foot square test section with cropped corners. The tunnel uses either air or a heavy gas as the test medium and can operate at stagnation pressures from near vacuum to atmospheric, has a Mach number range from near zero to 1.2 and is capable of maximum Reynolds numbers of about 3 million per foot in air and 10 million per foot in heavy gas. Prior to 1998 , the TDT used dichlorodifluoromethane, R-12, as the aerodynamic test medium. The TDT now uses 1,1,1,2 tetrafluoroethane, R-134a, as the test medium. ${ }^{11,12}$ The TDT is specially configured for flutter testing, with excellent model visibility from the control room and a rapid tunnel shutdown capability for model safety (bypass valves). Model mount systems include a sidewall turntable for semispan models, a variety of stings for full-span models, and a cable-mount system for "flying" models. The sidewall turntable was used for the current tests. The TDT also offers an airstream oscillation system for gust studies as well as digital controllers and supporting systems for active controls testing. Testing in heavy gas has important advantages over testing in air: improved model to full-scale similitude (which results in cheaper, heavier models with lower model elastic mode frequencies), higher Reynolds numbers, and reduced tunnel power requirements. These capabilities make the TDT the best-suited facility in the world for flutter testing large, full-span, aeroelastically-scaled models at transonic speeds.

\section{Oscillating Turntable (OTT)}

The OTT is a unique research tool at the TDT that provides the ability to oscillate relatively large, semispan wind-tunnel models in pitch at frequencies up to $40 \mathrm{~Hz}$. This research tool has been designed specifically for the acquisition of unsteady pressure

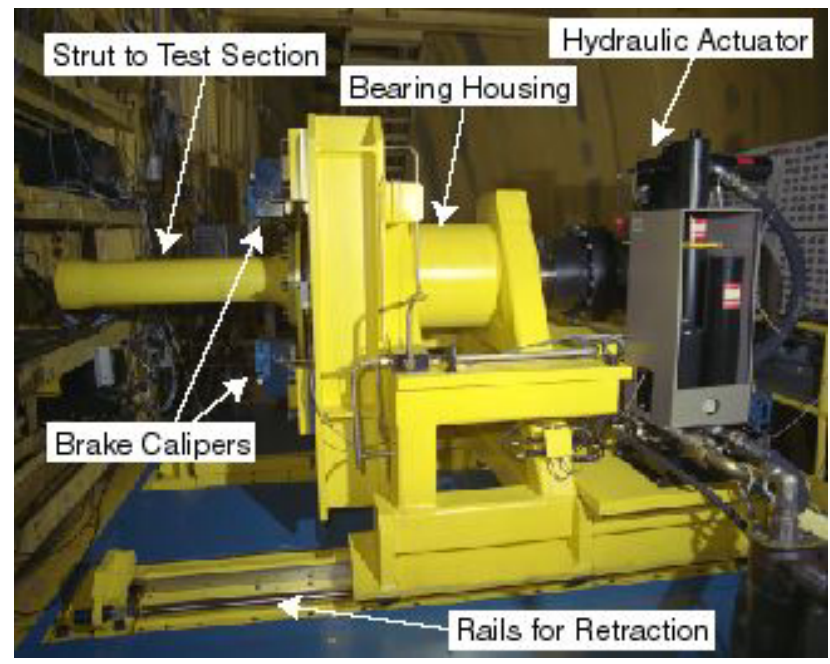

Fig. 1 Side view of the Oscillating Turntable (OTT).

and loads data on rigid wind-tunnel models in order to study flow phenomena associated with flutter, LCO, shock dynamics, and nonlinear unsteady aerodynamic effects on a wide variety of aerospace vehicle configurations at transonic speeds. ${ }^{2}$ Models may be oscillated sinusoidally at constant or varying frequencies, be subjected to a step input, or undergo user-defined motions. It is anticipated that unsteady pressure measurements due to precisely controlled model motions will provide valuable data for CFD correlation and aircraft design with respect to unsteady aerodynamic/aeroelastic phenomena.

Figure 1 highlights key components of the OTT. The OTT utilizes a powerful rotary hydraulic actuator, rated for 495,000 in-lbf, and a digital Proportional, Integral, Derivative, Feedforward (PIDF) control system to position and oscillate models. Power for the OTT is supplied by a 3000 psi, 150 gpm hydraulic power unit which is located outside the tunnel pressure shell. Rails allow for precise positioning of the OTT with respect to the tunnel wall to accommodate a wide range of models and model support systems. Cam wheels and clamps lock the OTT onto its rails. To minimize the exposure of model instrumentation, the wiring passes through a 2.5 inch diameter hole in the center of the entire OTT shaft and actuator.

\section{Rigid Semispan Model (RSM)}

The RSM planform is a $1 / 12$ th scale configuration based on an early design known as the Reference $\mathrm{H}$ configuration that was a component of the High Speed Research (HSR) program. ${ }^{4}$ Model airfoil shapes were based on those of the Reference $H$, with the model wing thickness being increased to a constant $4 \%$ thicknessto-chord ratio in order to accommodate pressure instrumentation at the wing tip. The model was designed to be very stiff to allow the measurement of aerodynamic properties with only negligible effects of 


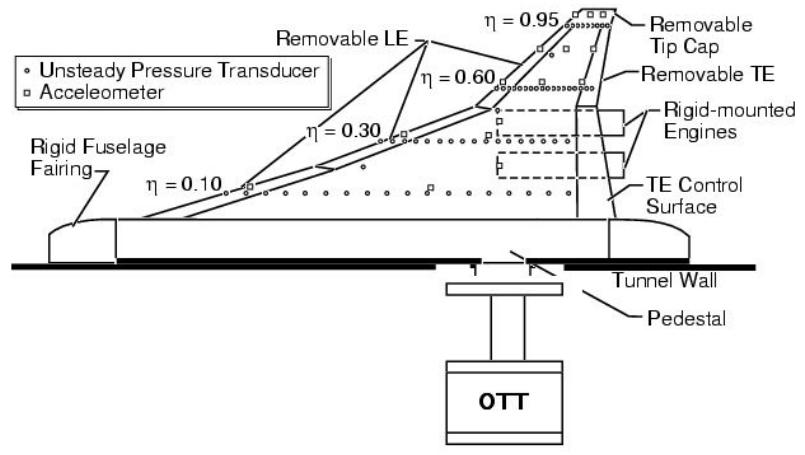

Fig. 2 Planform, model details, and instrumentation layout for the RSM wind-tunnel model.

structural deformations.

Figure 2 shows the planform layout and main components of the RSM including the OTT mount. The leading and trailing edges were removable in order to access pressure instrumentation in those regions. A removable tip cap allowed access to pressure instrumentation at the wing tip. The RSM could be tested either with or without a pair of flow-through nacelles. The nacelles were rigidly attached to pylons on the lower, inboard surface of the wing. The RSM wing had a graphite epoxy composite structure with an open-cell foam core. The RSM was re-built in 1995 after experiencing a failure of the bond of the upper and lower surfaces. Rivets were inserted along the front and rear spars to eliminate the possibility of a similar failure in future testing, and the original four-pound (i.e. a density of $4 \mathrm{lb} / \mathrm{ft}^{3}$ ) foam core was replaced with an eight-pound foam core for added strength and durability.

The RSM was tested with a rigid fuselage fairing which displaced the model away from the wind-tunnel wall boundary layer while serving as an aerodynamic boundary condition at the wing root. Additionally, the rigid fuselage fairing provided an aerodynamic shield for the hardware, instrumentation, and wire bundles located at the wing root. Two different fuselages were used with the RSM. A long fuselage fairing was used when it was tested on the balance and a shorter fuselage fairing was used when it was tested on the OTT. A photograph of the RSM, engine nacelles, and the 18-foot long fuselage fairing installed in the TDT test section is shown in figure 3 .

The instrumentation layout for the RSM (visible in figure 2) consisted of 131 insitu unsteady pressure transducers located at the 10, 30,60, and $95 \%$ span stations. Six additional unsteady pressure transducers were installed at the $20 \%$ chord station for the 20 , 45 , and $75 \%$ span stations for both upper and lower surfaces. Channels were carved into the foam core to

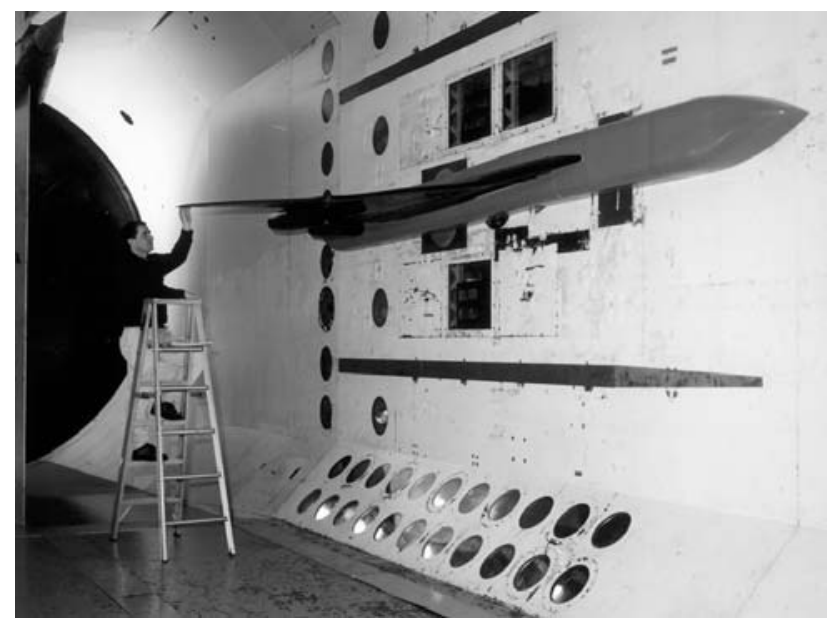

Fig. 3 The RSM and 18-foot fuselage mounted in the TDT test section.

accommodate the wiring for the instrumentation. Instrumentation also included accelerometers installed throughout the wing. The fuselage fairing used for testing the RSM on the OTT was instrumented with unsteady pressure transducers.

\section{Benchmark Supercritical Wing (BSCW)}

The BSCW is shown mounted in the TDT test section in Figure 4. The model has a rectangular planform with a 32 inch span, 16 inch chord, and a NASA SC(2)-0414 airfoil. Using 40 in-situ transducers, unsteady pressure measurements were made along the chord at the 60 percent spanwise location at Mach numbers ranging from 0.4 to 0.85 and $\mathrm{dy}^{-}$ namic pressures of 100,170 , and 200 psf in R-134a heavy gas. Reynolds numbers based on model chord ranged from 2.4 to 6.5 million and these test conditions corresponded to reduced frequencies $(\mathrm{k})$ from 0.011 to 0.579 for the BSCW (1 Hz to $30 \mathrm{~Hz})$. Boundary-layer transition was fixed at 7.5 percent chord and the OTT pitch axis was located at $x / c=0.3$. The BSCW model has been previously tested at the TDT as part of the Benchmark Models Program ${ }^{13,14}$ during which a large database of unsteady pressures were obtained during motions on a flexible pitch and plunge mount.

\section{Methodology}

Unsteady pressure measurements were made on the RSM and the BSCW while the models underwent pitch oscillations on the OTT at frequencies from 1 to 10 $\mathrm{Hz}$ (RSM) and from 1 to $30 \mathrm{~Hz}$ (BSCW). In addition, unsteady pressures were acquired during RSM/OTT and BSCW/OTT step inputs in order to provide data to compute aerodynamic impulse responses.

The identification of aerodynamic impulse responses can be performed using system identification techniques. The computational identification of unsteady aerodynamic impulse responses in order to develop efficient CFD-based models for aeroelastic analyses has recently been performed. ${ }^{10}$ These efficient compu- 


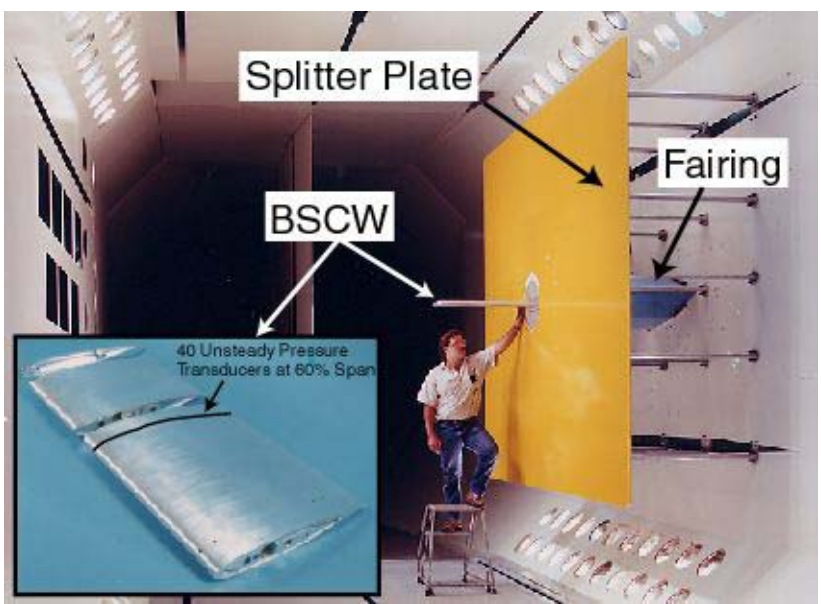

Fig. 4 The Benchmark Supercritical Wing windtunnel model.

tational models are known as reduced-order models (ROMs) and comprise one aspect of a very active research effort at several research organizations. ${ }^{9}$

The identification of experimental unsteady aerodynamic (pressure) ROMs can be performed by using the same techniques used to identify the computational unsteady aerodynamic ROMs. The Volterra theory of nonlinear systems is used as the basis for modeling the linear and nonlinear dynamic response of the unsteady aerodynamic system under investigation. The basic premise of the Volterra theory is that the response of a nonlinear system to an arbitrary input can be approximated by the infinite sum of multidimensional convolution integrals

$\mathrm{y}(\mathrm{t})=\int \mathrm{h}_{1}(t-\tau) u(\tau) d \tau+\iint \mathrm{h}_{2}\left(t-\tau_{1}, t-\right.$ $\left.\tau_{2}\right) u\left(\tau_{1}, \tau_{2}\right) d \tau_{1} d \tau_{2}+\ldots$

The kernels $\left(h_{1}, h_{2}, \ldots\right)$ represent various linear and nonlinear levels of the dynamics of the system. The first-order kernel $\left(h_{1}\right)$ is the linear (or linearized) impulse response and the higher-order kernels $\left(h_{2}\right.$ and higher) represent second-order nonlinear impulse responses, third-order nonlinear impulse responses, and so on.

For the present study, the identification of experimental unsteady aerodynamic impulse responses will be limited to the first-order, or linearized, kernel. It is referred to as a linearized kernel since identification of the kernel (impulse response) may occur about a nonlinear steady state condition (such as a transonic Mach number). Future research will focus on the identification of the second-order kernel. The frequency-domain version of the second-order kernel is known as the bispectra, which has found important applications across a wide variety of disciplines for quantifying experimental nonlinear dynamics. ${ }^{15}$

The identification of the experimental unsteady aerodynamic impulse responses (first-order kernel) will consist of the deconvolution of a given input/output pair. Deconvolution involves the extraction of the impulse response of a system when the input and corresponding output are known. The input, in this case, is a sequence of positive and negative step inputs in pitch applied using the OTT and the output is any of several measured pressure responses from the windtunnel models. Deconvolution is then used to extract the impulse response for the given input/output pair. For the given OTT step input, an impulse response can be identified for each pressure measurement (sensor) on the wind-tunnel models.

Once the impulse response has been generated, convolution is used to predict the pressure response due to sinusoidal inputs in pitch at various frequencies. The measured results are compared to the predicted results (via convolution) in the next section.

\section{RSM/OTT Results}

Results for the RSM/OTT are presented in this section. The RSM has a total of 131 in-situ pressure transducers. A step input in pitch using the OTT results in 131 unsteady pressure responses due to the step input in pitch. Therefore, deconvolution can be applied to all of the unsteady pressure measurements to yield 131 unsteady aerodynamic impulse responses. For the sake of brevity and to demonstrate the feasibility of the method, results are presented for only one pressure measurement located on the upper surface of the RSM at the $60 \%$ span location and the $30 \%$ chord station. The data was acquired at a Mach number (M) of 0.8 , a dynamic pressure (q) of $150 \mathrm{psf}$, and with the RSM at zero degrees angle of attack.

Figure 5 presents the step pitch input commanded to the OTT and the resultant pressure response at the pressure transducer location mentioned above. Although a theoretical step input consists of an infinite slope where the step occurs, a physically realizable step input, such as that commanded by the OTT, will be limited by the pitch inertia, stress, and load limitations of the model undergoing pitch. As can be seen, a step input that closely approaches a theoretical step input can, in fact, be applied by the OTT. The unsteady pressure measurement is also quite noisy, as can be seen.

Using the sequence of step pitch motions of the OTT as the input and the unsteady pressure measurement as the output, deconvolution is applied to identify the unsteady aerodynamic impulse response. Figure 6 presents the time- and frequency-domain versions of the pressure impulse response identified via deconvolution. As can be seen in Figure 6(b), the identified impulse response exhibits significant frequency content, as is to be expected for an impulse response. An analysis of the unsteady aerodynamic impulse responses at all pressure transducer locations can provide a spatial mapping of the frequency characteristics of a given configuration at a given test condition. This type of 


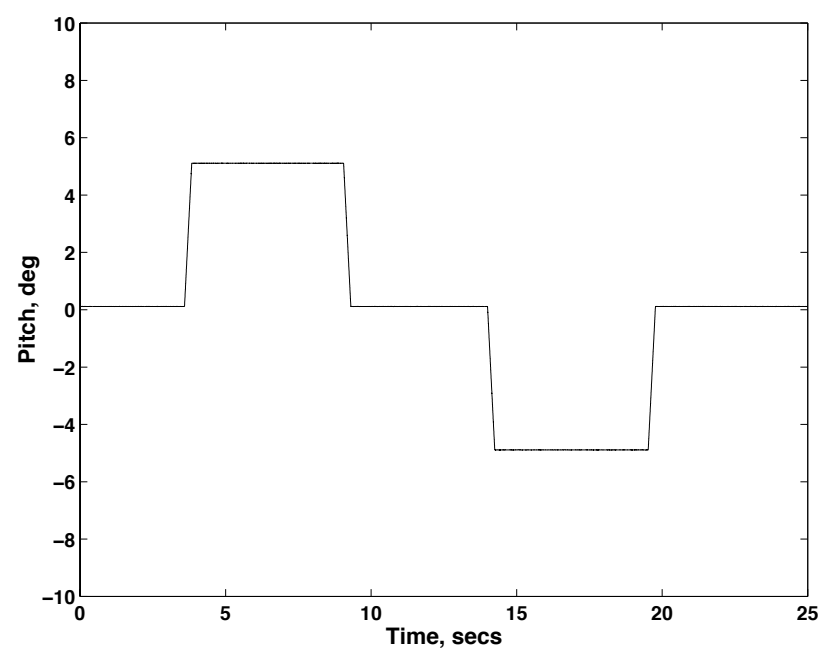

a) Step Input in Pitch

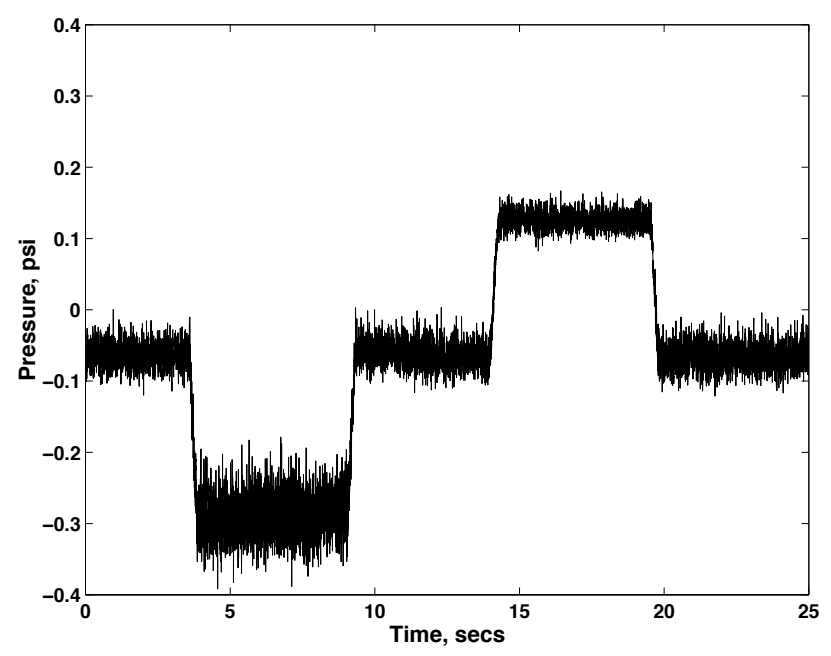

b) Pressure Response due to Step Input in Pitch

Fig. 5 Commanded pitch motion and resultant pressure response on the upper surface of the RSM at $60 \%$ span and $30 \%$ chord at $M=0.8, q=150$ psf.

spatial mapping may be useful for the design and optimal placement of various flow control devices.

Upon identification, the unsteady aerodynamic impulse response can then be used to predict the unsteady aerodynamic response due to any OTT input using convolution and the impulse response of Figure 6 . In the following figures, comparisons are made between predicted unsteady aerodynamic responses and the measured responses for several sinusoidal OTT motions.

Figure 7 presents the comparison between the measured pressure response and the corresponding predicted pressure response for a commanded oscillation of $1.2 \mathrm{~Hz}$. The comparison is excellent and demonstrates the ability of the method to capture the dominant (driving) frequency while filtering out uncorrelated noise. The deconvolution process automatically identifies the input/output correlations which results

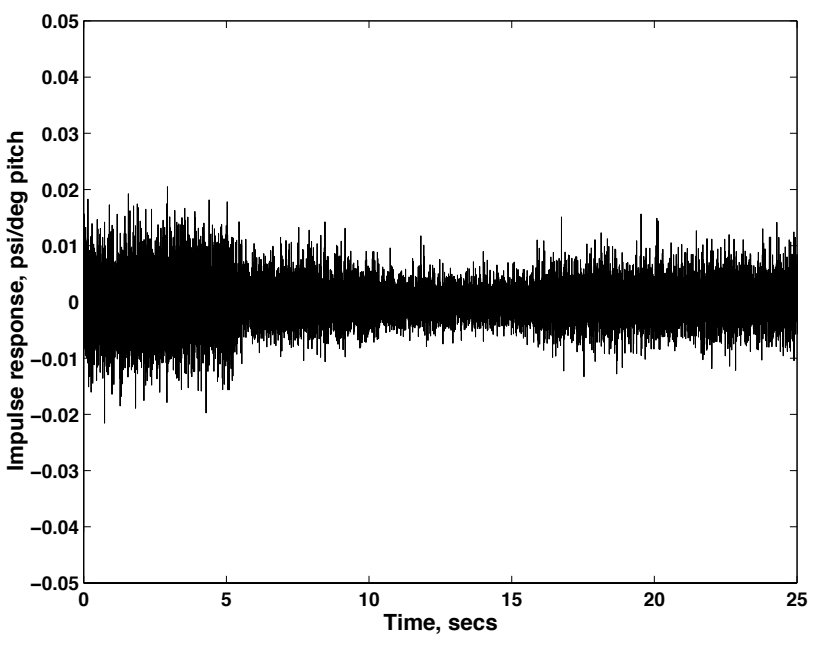

a) Time domain

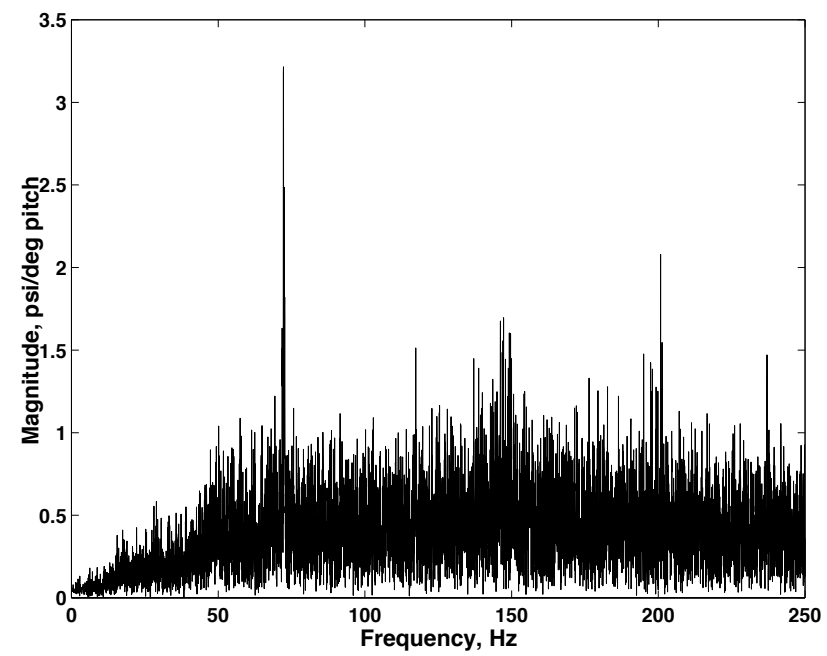

b) Frequency domain

Fig. 6 Pressure impulse response obtained via deconvolution for the RSM; time domain and frequency domain (magnitude).

in the impulse response. The process of identifying these correlations for a given input/output pair also has the added benefit that it filters out any information that is not correlated to the input. Therefore, uncorrelated measurement noise, for example, is automatically removed as the impulse response is generated. This filtering capability is visible in Figure 7(b).

Figure 8 presents the comparison between the measured pressure response and the corresponding predicted pressure response for a commanded oscillation of $5.1 \mathrm{~Hz}$. Again, the method clearly identifies the correct frequency component for this pressure measurement along with good filtering of the noise.

Figure 9 presents the comparison between the measured pressure response and the corresponding predicted pressure response for a commanded oscillation of $8.2 \mathrm{~Hz}$. At this frequency, some higher-frequency content begins to appear in the predicted response as 


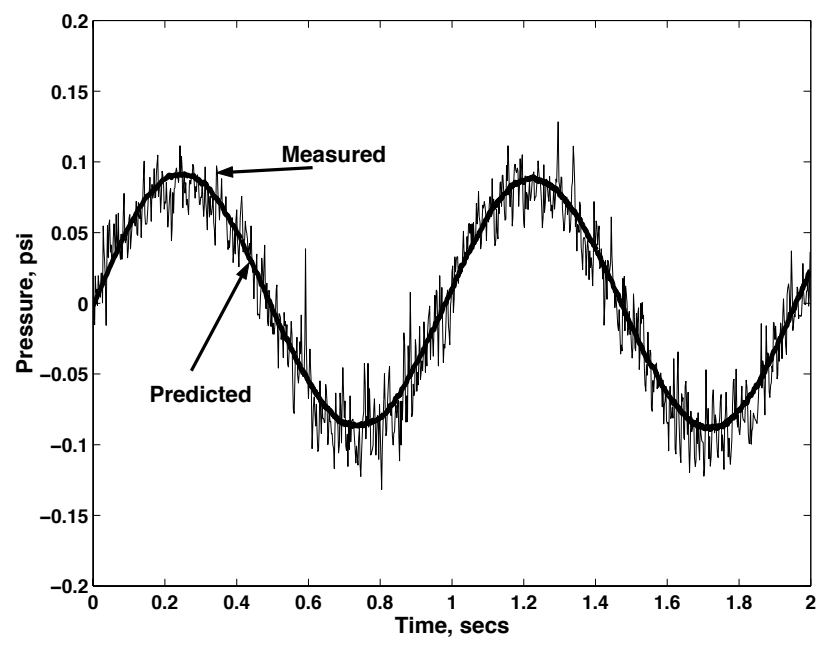

a) Time domain

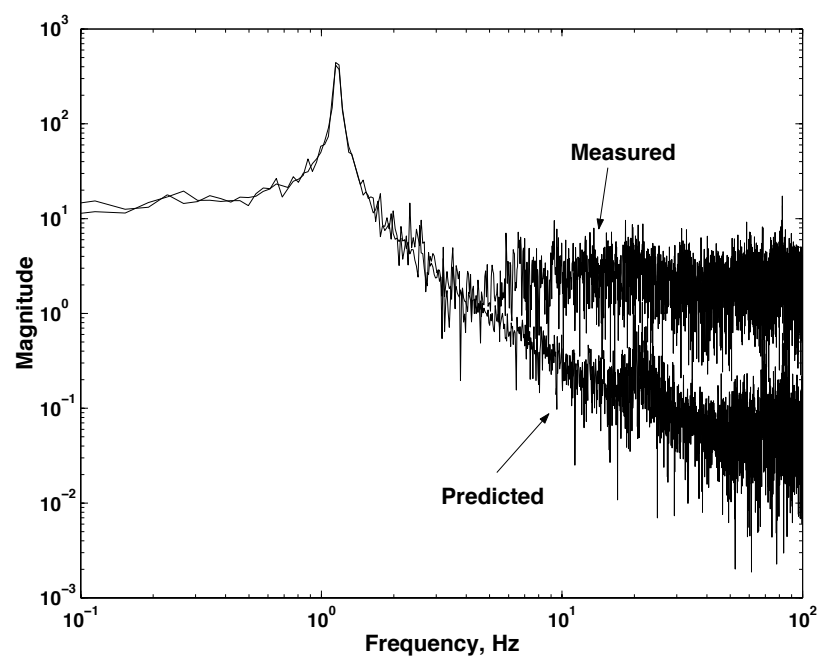

b) Frequency domain

Fig. 7 Measured and predicted pressure responses due to a $1.2 \mathrm{~Hz}$ sinusoidal motion of the OTT for the RSM; time domain and frequency domain (magnitude).

well.

Figure 10 presents the comparison between the measured pressure response and the corresponding predicted pressure response for a commanded oscillation of $10.0 \mathrm{~Hz}$. For this case, without the predicted response, it would be very difficult to discern any periodicity in the measured response. The filtering capability of the deconvolution method proves to be essential at this frequency.

At this condition, the linearity of the measured pressure responses (for this pressure transducer location) is defined by the excellent correlation between the experimental results and the results computed using convolution. If predicted results do not compare well with measured results, this could be an indication that some nonlinearity has influenced the measured response.

In addition, because deconvolution involves in-

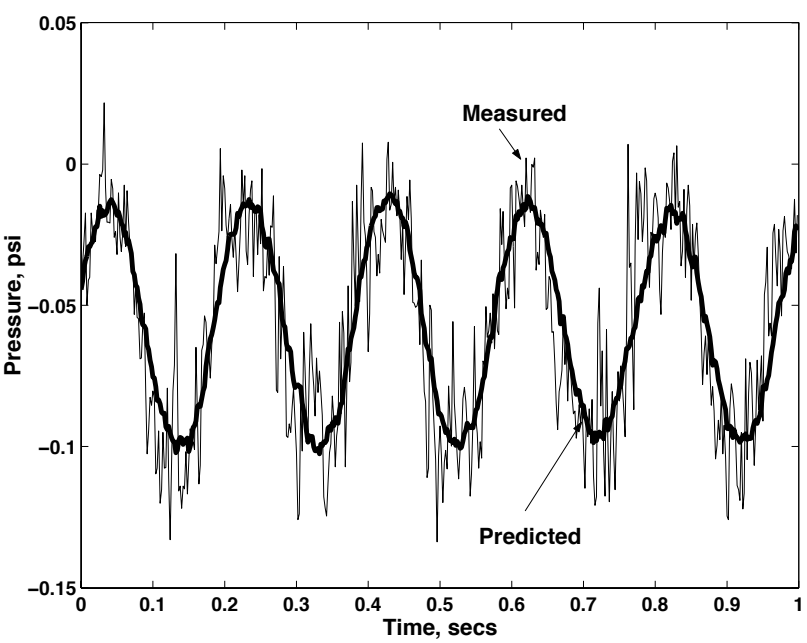

a) Time domain

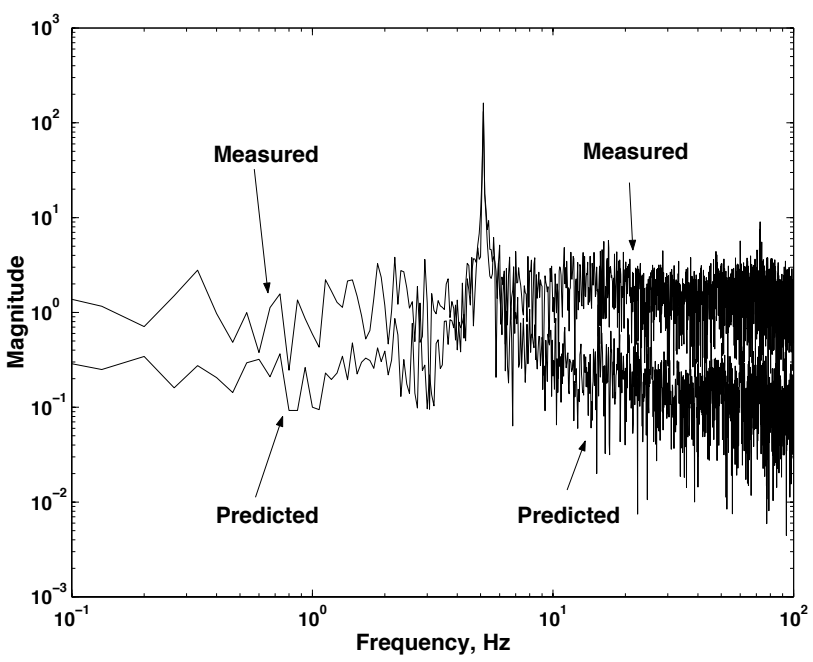

b) Frequency domain

Fig. 8 Measured and predicted pressure responses due to a $5.5 \mathrm{~Hz}$ sinusoidal motion of the OTT for the RSM; time domain and frequency domain (magnitude).

put/output correlation, any uncorrelated white noise (measurement noise) is easily filtered out. Note that for several of the examples presented, the filtering was applied at all uncorrelated frequencies, both low and high frequencies. Simple low-pass or high-pass filters would not be able to match this level of filtering capability and much more sophisticated band-pass filters would have to be introduced. However, even with band-pass filters, the question of which frequency range to filter would remain a serious question for the analyst. Deconvolution automatically handles the filtering without a priori definition of a frequency range where filtering is desired. Analysis of these results can subsequently be used to identify regions of linear and nonlinear behavior which will be helpful in understanding dominant flow physics. 


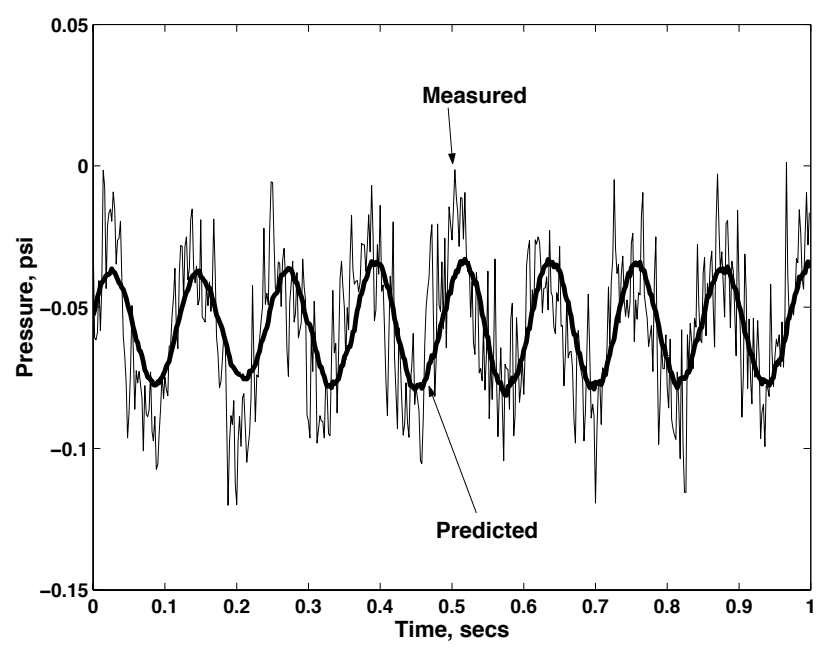

a) Time domain

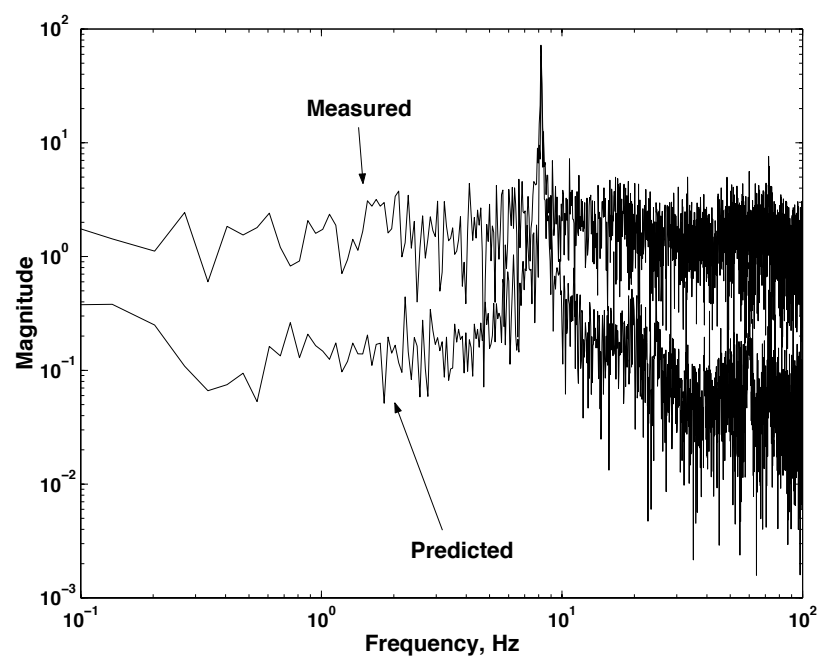

b) Frequency domain

Fig. 9 Measured and predicted pressure responses due to a $8.2 \mathrm{~Hz}$ sinusoidal motion of the OTT for the RSM; time domain and frequency domain (magnitude).

\section{BSCW/OTT Results}

For the BSCW on the OTT, results are presented for pressure measurements at the trailing edge, at a Mach number of 0.5 , a dynamic pressure of $100 \mathrm{psf}$, and zero degrees angle of attack. Figure 11 presents the commanded step input in pitch applied to the OTT (input) and the corresponding pressure response (output). This input/output pair is then used in a deconvolution process to extract the corresponding pressure impulse response.

Presented in Figure 12 is the unsteady aerodynamic impulse response identified via deconvolution, both in the time and frequency domain. From Figure 12(b), the response (for this condition and this position on the airfoil) appears fairly flat at lower frequencies with increasing content at higher frequencies. Again, comparison of unsteady aerodynamic impulse response

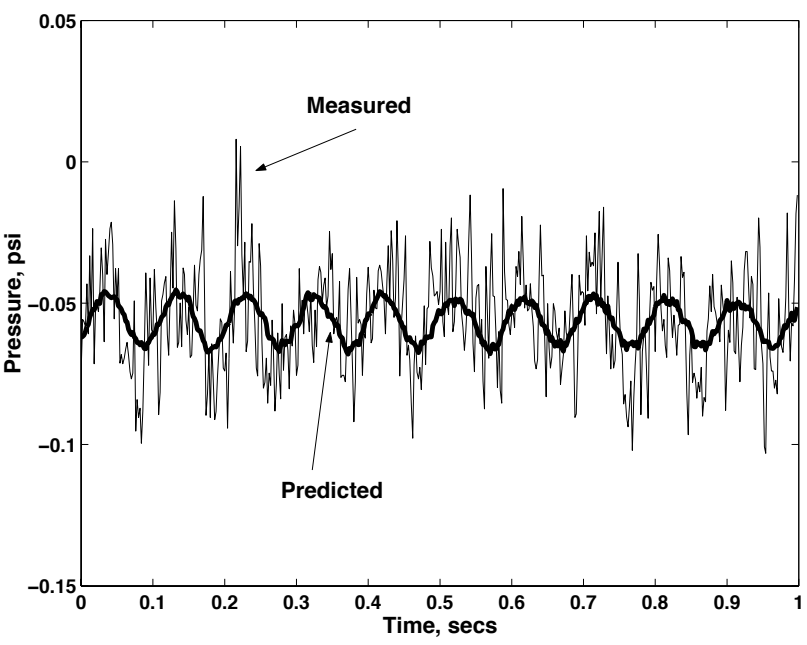

a) Time domain

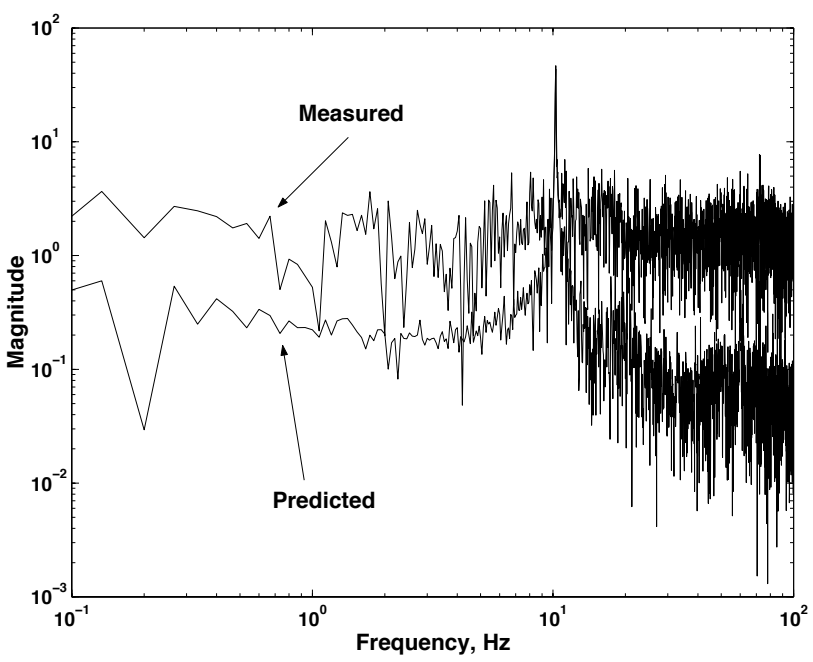

b) Frequency domain

Fig. 10 Measured and predicted pressure responses due to a $10.1 \mathrm{~Hz}$ sinusoidal motion of the OTT; time domain and frequency domain (magnitude).

functions for a given configuration at several conditions and at several pressure ports may be useful in identifying dominant flow physics and the frequency range at which these phenomena occur. The important point to be made in this paper is that these functions (unsteady aerodynamic impulse responses) exist and can be identified for any configuration.

Figure 13 presents the measured and predicted pressure responses due to a $1 \mathrm{~Hz}$ sinusoidal motion of the OTT in the time domain and frequency domain (magnitude). Again, the comparison is very good and demonstrates the ability of the method to capture the dominant (driving) frequency while filtering out uncorrelated noise. Once again, the filtering is applied uniformly to both low and high uncorrelated frequencies.

Figure 14 presents the measured and predicted pres- 


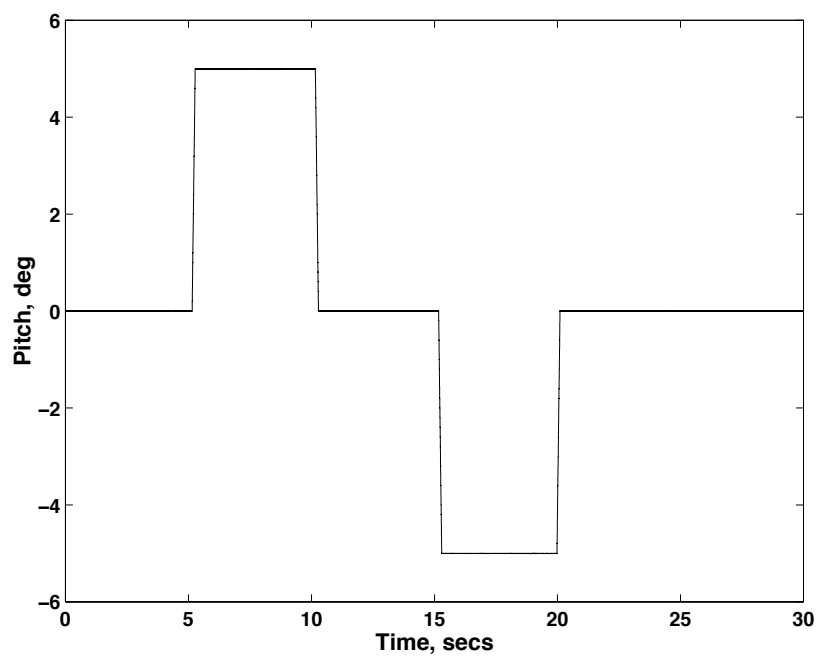

a) Step Input in Pitch

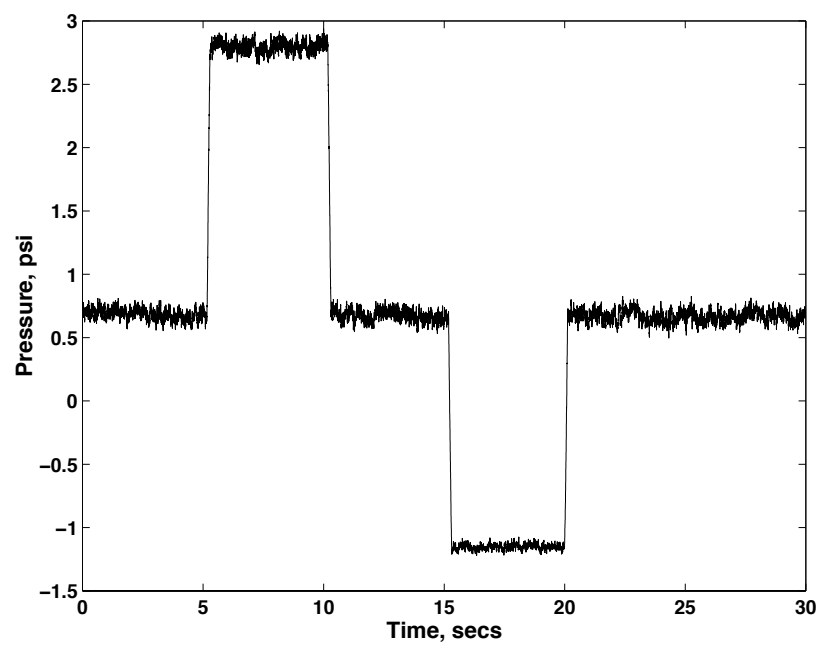

b) Pressure Response due to Step Input in Pitch

Fig. 11 Commanded pitch motion and resultant pressure response at the trailing edge at $\mathrm{M}=\mathbf{0 . 5}$, $\mathrm{Q}=100 \mathrm{psf}$ for the BSCW.

sure responses due to a $2 \mathrm{~Hz}$ sinusoidal motion of the OTT in the time domain and frequency domain (magnitude). The comparison is very good with excellent filtering of the uncorrelated noise.

Finally, Figure 15 presents the measured and predicted pressure responses due to a $15 \mathrm{~Hz}$ sinusoidal motion of the OTT in the time domain and frequency domain (magnitude). For this case the comparison is good but Figure 15(a) indicates an asymmetry in the measured response not captured by the predicted response. Additional analyses are required to determine if this difference is due to a possible nonlinear effect. Although at this subsonic Mach number nonlinear aerodynamic effects are not expected in general, since this pressure measurement is at the trailing edge, some local flow separation induced by the higher frequency may be occuring.

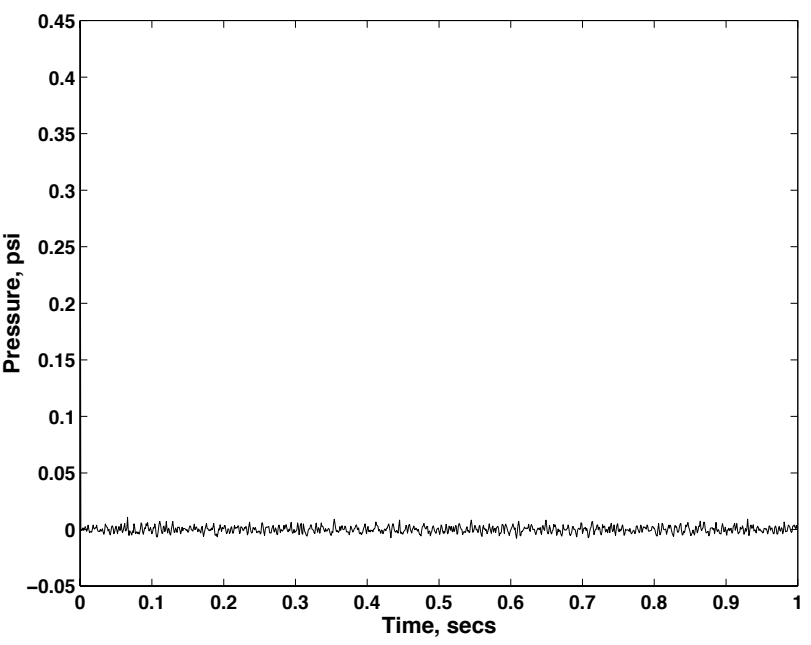

a) Time domain

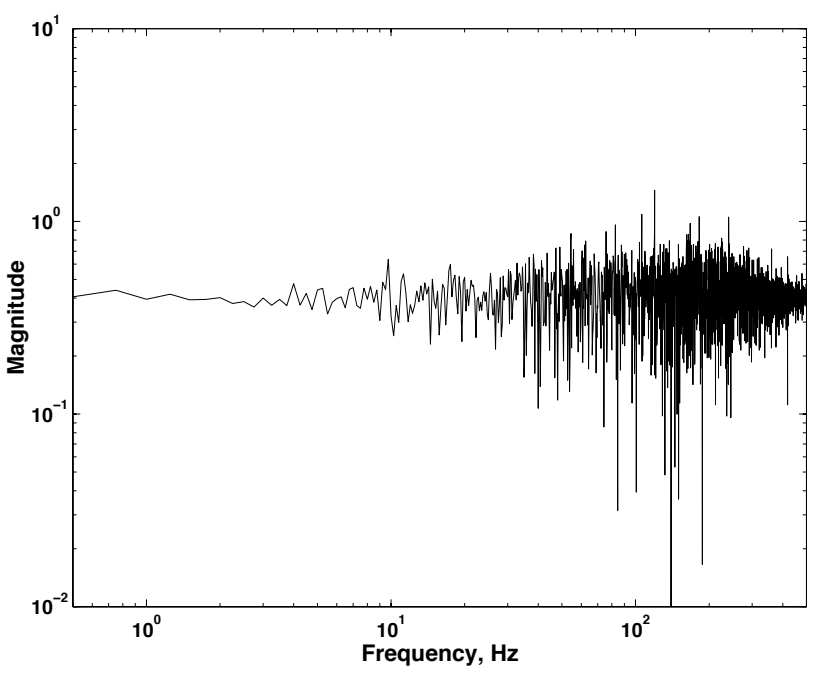

b) Frequency domain

Fig. 12 Pressure impulse response obtained via deconvolution for the BSCW at the trailing edge; time domain and frequency domain (magnitude).

\section{Concluding Remarks}

A method for the identification of experimental aerodynamic impulse responses has been presented. The results verify the existence of these functions in an experimental setting. The results presented included applications of the method to unsteady aerodynamic (pressure) responses for two wind-tunnel models: a Rigid Semispan Model (RSM) and a Benchmark Supercritical Wing (BSCW). The method was used to successfully predict the pressure responses due to various sinusoidal oscillations for both wind-tunnel models. An added bonus of the method is its ability to filter out uncorrelated white noise from noisy measurements. The method can, therefore, be used to 1) identify the level of linearity for a given measurement and 2) filter out the noise from experimental measurements. Additional analyses are required to 1) further investigate the value of spatial frequency mappings for 


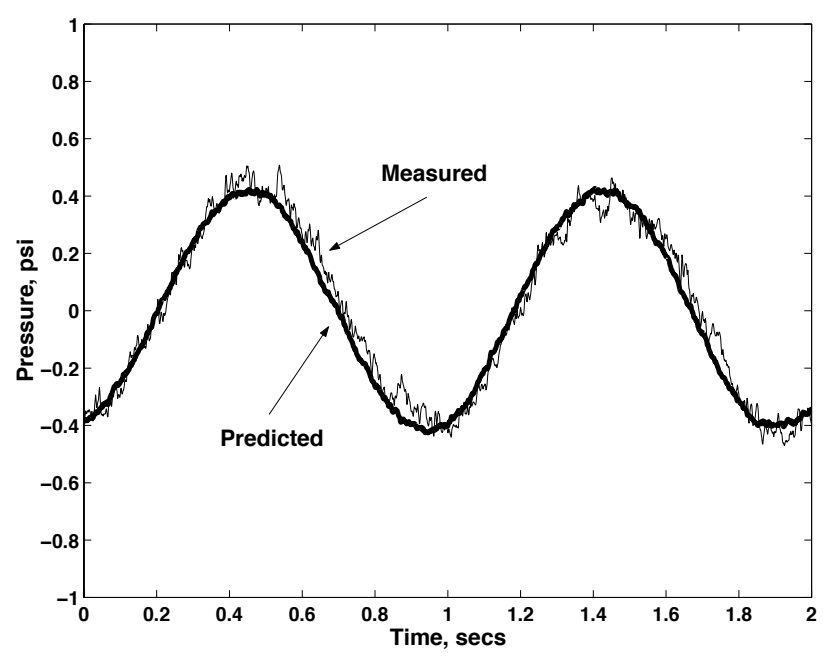

a) Time domain

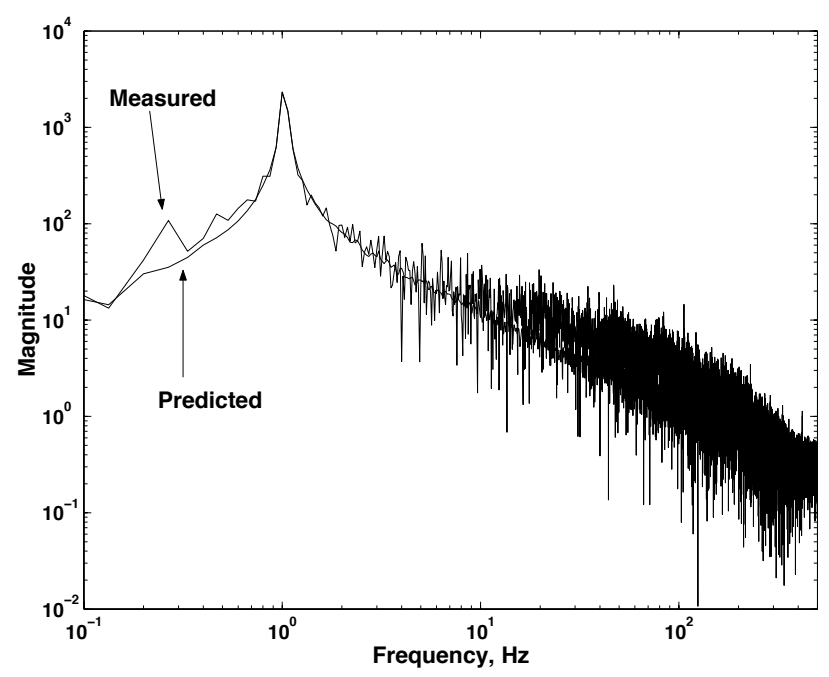

b) Frequency domain

Fig. 13 Measured and predicted pressure responses due to a $1 \mathrm{~Hz}$ sinusoidal motion of the OTT for the BSCW; time domain and frequency domain (magnitude).

a given configuration and 2) use the information provided by this method to gain insight into the dominant flow physics. These results represent the first time that experimental unsteady aerodynamic impulse responses have been successfully identified.

\section{References}

${ }^{1}$ Silva, W. A., Keller, D. F., Florance, J. R., Cole, S. R., and Scott, R. C., "Experimental Steady and Unsteady Aerodynamic and Flutter Results for HSCT Semispan Models," AIAA/ASME/ASCE/AHS/ASC 41st Structures, Structural Dynamics, and Materials Conference, No. 2000-1697, April 2000.

${ }^{2}$ Piatak, D. J. and Cleckner, C. S., "A New Forced Oscillation Capability for the Transonic Dynamics Tunnel," $40 t h$ Aerospace Sciences Meeting and Exhibit, Reno, NV, Jan. 2002.

${ }^{3}$ Murphy, P. C. and Klein, V., "Estimation of Aircraft Unsteady Aerodynamic Parameters from Dynamic Wind Tunnel Testing," AIA A Atmospheric Flight Mechanics Conference and Exhibit, No. 01-2975, Montreal, Canada, Aug. 2001.

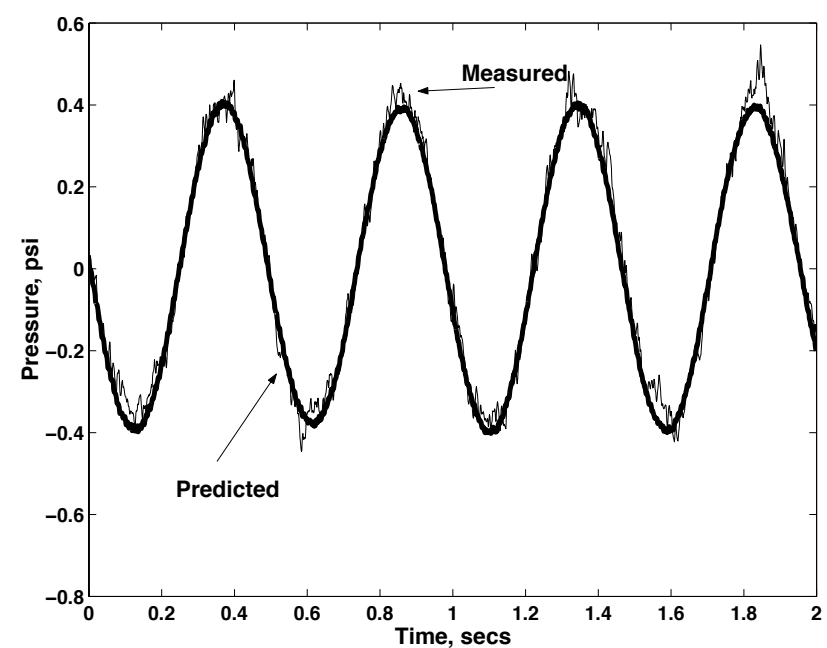

a) Time domain

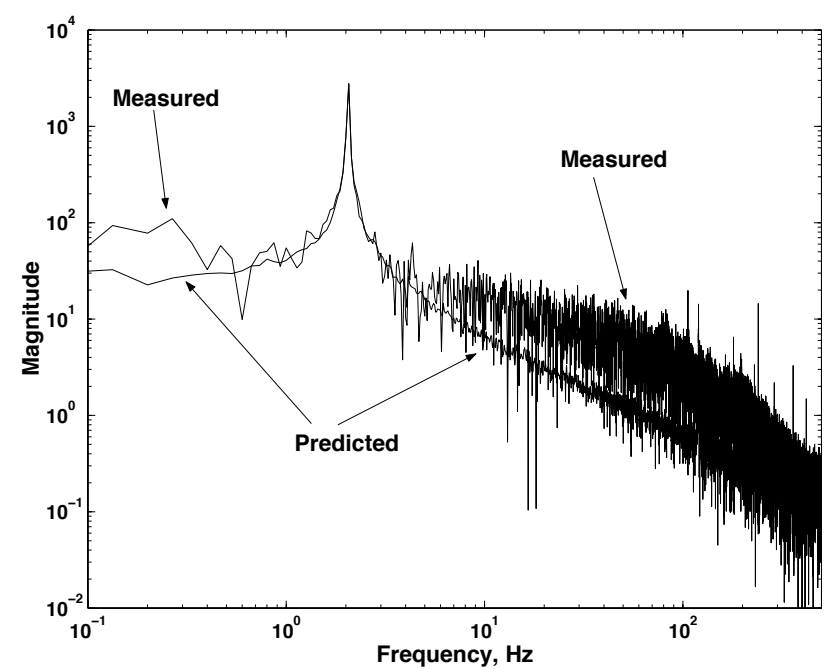

b) Frequency domain

Fig. 14 Measured and predicted pressure responses due to a $2 \mathrm{~Hz}$ sinusoidal motion of the OTT for the BSCW; time domain and frequency domain (magnitude).

${ }^{4}$ Scott, R. C., Silva, W. A., Florance, J. R., and Keller, D. F., "Measurement of Unsteady Pressure Data on a Large HSCT Semispan Wing and Comparison with Analysis," Proceedings of the $43 \mathrm{rd}$ AIAA/ASME/ASCE/AHS/ASC Structures, Structural Dynamics, and Materials Conference, No. 02-1648, Denver, CO, April 2002.

${ }^{5}$ Parekh, D. E. and Glezer, A., "AVIA: Adaptive Virtual Aerosurface," Fluids 2000 Conference and Exhibit, No. 00-2474, Denver, CO, June 2000.

${ }^{6}$ Honohan, A. M., Amitay, M., and Glezer, A., "AVIA: Adaptive Virtual Aerosurface," Fluids 2000 Conference and Exhibit, No. 00-2401, Denver, CO, June 2000.

${ }^{7}$ Chatlynne, E., Rumigny, N., Amitay, M., and Glezer, A., "Virtual Aero-Shaping of a Clark-Y Airfoil Using Synthetic Jet Actuators," 39th Aerospace Sciences Meeting and Exhibit, No. 01-0732, Reno, NV, Jan. 2001.

${ }^{8}$ Amitay, M., Horvath, M., Michaux, M., and Glezer, A., "Virtual Aerodynamic Shape Modification at Low Angles of Attack using Synthetic Jet Actuators," 31st AIA A Fluid Dynamics Conference and Exhibit, No. 01-2975, Anaheim, CA, June 2001.

${ }^{9}$ Beran, P. S. and Silva, W. A., "Reduced-Order Modeling: 


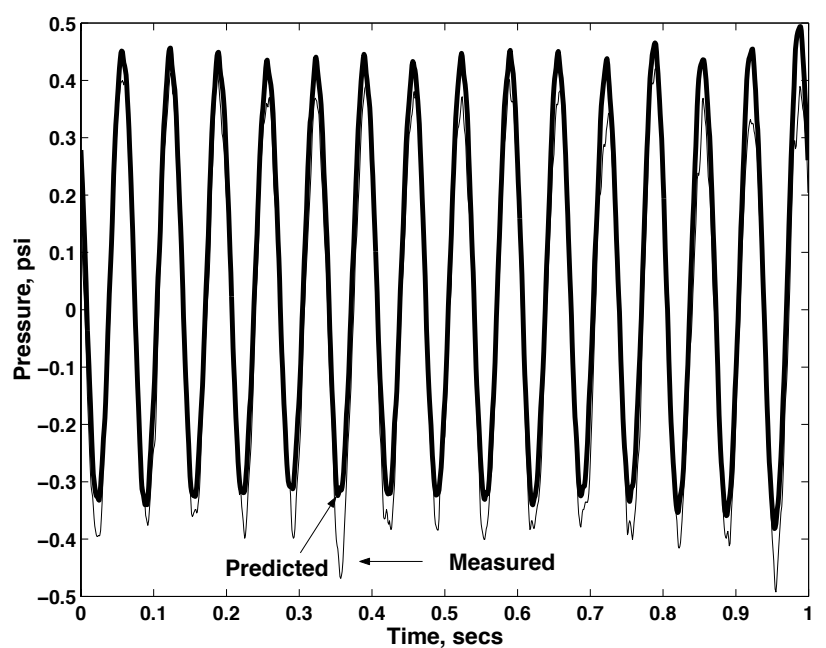

a) Time domain

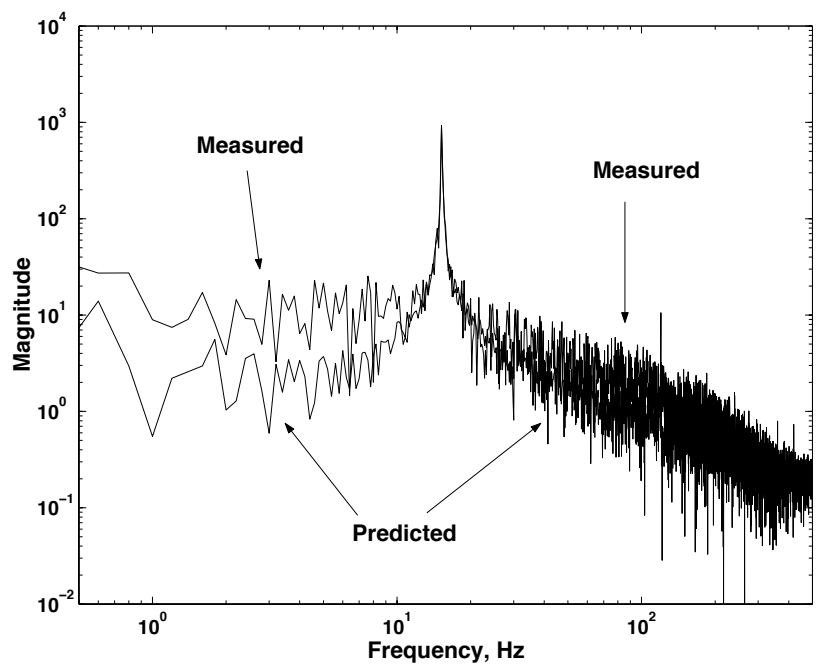

b) Frequency domain

Fig. 15 Measured and predicted pressure responses due to a $15 \mathrm{~Hz}$ sinusoidal motion of the OTT for the BSCW; time domain and frequency domain (magnitude).

New Approaches for Computational Physics," Presented at the $39 t h$ AIAA Aerospace Sciences Meeting, 8-11 January 2001, Reno, NV, January 2001.

${ }^{10}$ Silva, W. A. and Bartels, R. E., "Development of ReducedOrder Models for Aeroelastic Analysis and Flutter Prediction Using the CFL3Dv6.0 Code," Proceedings of the 43rd Structures, Structural Dynamics and Materials Conference, No. 021596, Denver, CO, April 2002.

${ }^{11}$ Corliss, J. M. and Cole, S. R., "Heavy Gas Conversion of the NASA Langley Transonic Dynamics Tunnel," Proceedings of the 20th Advanced Measurements and Ground Testing Technology Conference, No. 98-2710, Albuquerque, NM, June 1998.

${ }^{12}$ Cole, S. R. and Rivera Jr, J. A., "The New Heavy Gas Testing Capability in the NASA Langley Transonic Dynamics Tunnel," Royal Aeronautical Society Wind Tunnels and Wind Tunnel Test Techniques Forum, No. No. 4, Cambridge, UK, April 1997.

${ }^{13}$ Bennett, R. M., Eckstrom, C. V., Rivera, J. A., Dansberry, B. E., Farmer, M. G., and Durham, M. H., "The Benchmark Aeroelastic Models Program-Description and Highlights of Initial Results," NASA TM-104180, Dec. 1991.
${ }^{14}$ Dansberry, B. E., Durham, M. H., Bennett, R. M., Rivera, J. A., Silva, W. A., and Wieseman, C. D., "Experimental Unsteady Pressures at Flutter on the Supercritical Wing Benchmark Model," AIA A/ASME/ASCE/AHS/ASC 34 th Structures, Structural Dynamics, and Materials Conference, La Jolla, CA, April 1993.

${ }^{15}$ Hajj, M. R. and Silva, W. A., "Nonlinear Flutter Aspects of the Flexible HSCT Semispan Model," Proceedings of the 44 th Structures, Structural Dynamics and Materials Conference, No. 2003-1515, Norfolk, VA, April 2003. 